\title{
Multiple Vertebral Hemangiomas Mimicking Metastases in a Patient with Renal Carcinoma: Importance of Hybrid Imaging with SPECT/CT and FDG PET/CT
}

\author{
K Manohar, RVR Parghane, S Kumari, A Bhattacharya, AK Mandal, BR Mittal
}

\begin{abstract}
Vertebral hemangiomas are the most common benign tumors of the spine and usually present as cold defects on skeletal scintigraphy. However, these lesions may rarely show uptake of Tc-99m methylene diphosphonate, mimicking metastases. In this patient with renal carcinoma, abnormal vertebral tracer uptake (indicative of skeletal metastases on planar bone scintigraphy) was subsequently localized to vertebral lesions identified as hemangiomas on the CT component of single photon emission computed tomography/computed tomography (SPECT/CT). None of these hemangiomas showed any uptake of F-18 fluorodeoxyglucose on positron emission tomography/ computed tomography (PET/CT).
\end{abstract}

Keywords: Vertebral hemangioma, ${ }^{99 \mathrm{~m}} \mathrm{Tc}-\mathrm{MDP}, \mathrm{SPECT/CT}$, F-18 FDG PET/CT.

How to cite this article: Manohar K, Parghane RVR, Kumari S, Bhattacharya A, Mandal AK, Mittal BR. Multiple Vertebral Hemangiomas Mimicking Metastases in a Patient with Renal
Carcinoma: Importance of Hybrid Imaging with SPECT/CT and FDG PET/CT. J Postgrad Med Edu Res 2012;46(4):196-197.

Source of support: Nil

Conflict of interest: None declared

\section{CASE REPORT}

A 64-year-old male patient diagnosed with left sided renal cell carcinoma underwent whole body bone scintigraphy (Fig. 1A) with Tc-99m methylene diphosphonate (MDP) to assess for any skeletal metastases. Increased tracer uptake suggestive of metastatic skeletal involvement was seen in the thoracic (D8) and lumbar (L4) vertebrae. Single photon emission computed tomography/computed tomography (SPECT/CT) images (Figs 1B, C, E and F) showed that MDP uptake in the D8 vertebra was localized to a typical hemangioma with vertical trabecular thickening identified

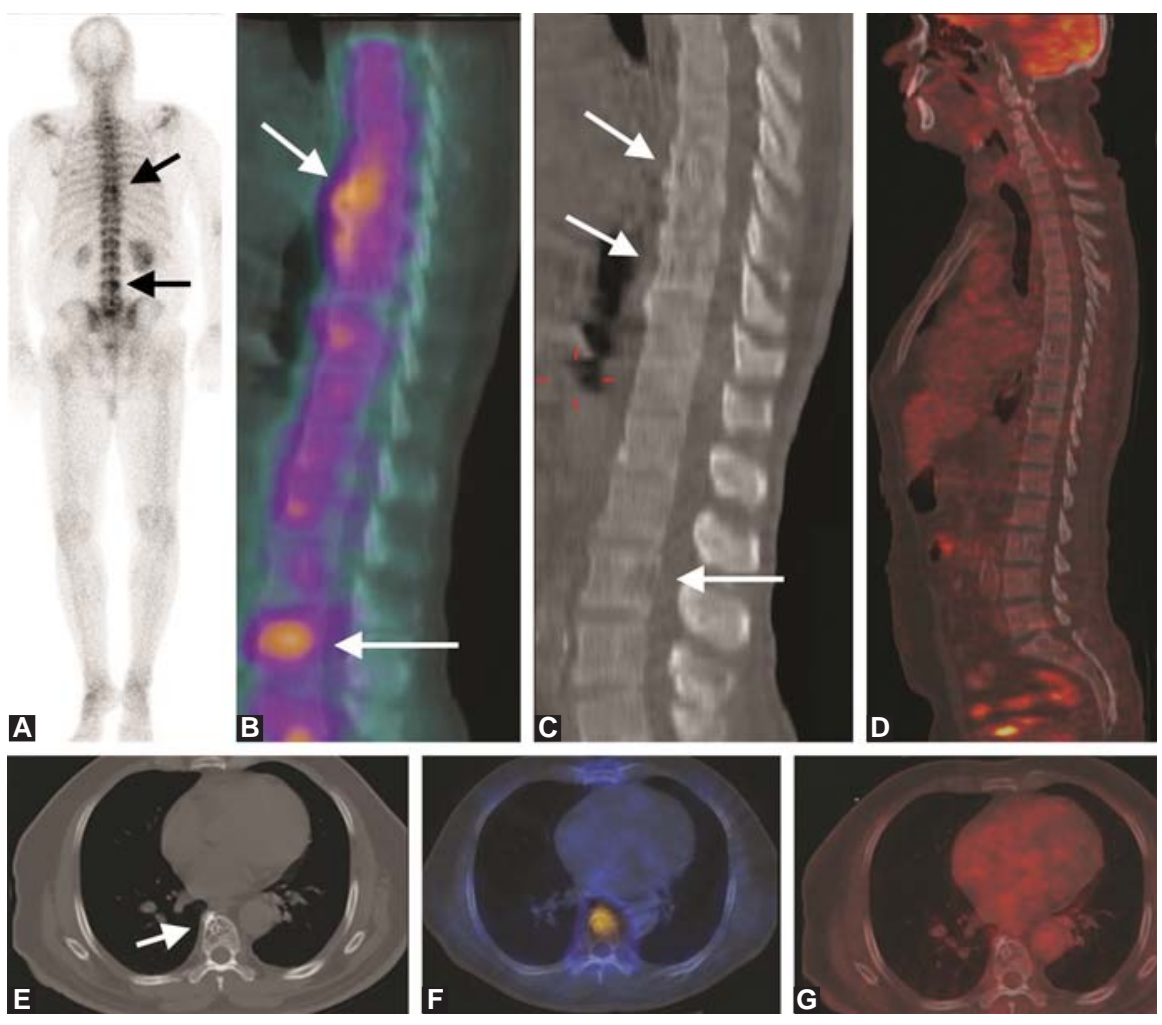

Figs 1A to G: (A) Whole body Tc-99m methylene diphosphonate (MDP) bone scan showing increased tracer uptake in the thoracic (D8) and lumbar (L4) vertebrae (arrows), (B) fused sagittal single photon emission computed tomography/computed tomography (SPECT/CT) image showing increased MDP uptake in the D8 and L4 vertebrae (arrows), (C) sagittal CT image showing vertical trabecular thickening in the D8, D10 and L3 vertebrae (arrows), (D) sagittal PET/CT image showing absence of FDG uptake in the vertebral column, (E) axial CT image showing trabecular thickening in the D8 vertebra (arrow), (F) fused transaxial SPECT/CT image showing increased MDP uptake in the D8 vertebra, (G) axial PET/CT image showing absence of FDG uptake in the D8 vertebra 
on the CT images. The posterior half of the body of the L4 vertebra showed MDP avidity with intralesional trabecular thickening suggestive of a hemangioma. Review of the CT images of the thoracolumbar spine (Fig. 1C) also revealed similar lesions in the D10 and L3 vertebrae. However, these hemangiomas did not show any MDP uptake. MDP uptake was also noted in degenerative disk changes at the lumbosacral (L5-S1) junction. The patient subsequently underwent F-18 fluorodeoxyglucose positron emission tomography/computed tomography (FDG PET)/CT), which did not reveal FDG uptake in any of the lesions (Figs 1D and $G$ ), ruling out metastatic involvement. Intense FDG uptake (SUVmax 7.2) was seen in the renal mass with moderate uptake (SUVmax 3.4) in a few retroperitoneal lymph nodes (not shown here).

\section{DISCUSSION}

Skeletal scintigraphy with MDP remains an essential investigation in the metastatic work up of various malignancies including renal cell carcinoma. However, the nonspecific accumulation of MDP in conditions other than metastases impairs the specificity of this technique. The fusion of anatomical information provided by CT images may resolve this problem. The utility of SPECT/CT in the interpretation of indeterminate lesions on planar bone scans has been demonstrated in many studies. ${ }^{1-4}$ Hemangiomas are the most common benign tumors of the spine and are most commonly located in the thoracic and lumbar spine followed by the craniofacial bones. ${ }^{5}$ A vertebral hemangioma usually presents as a photopenic 'cold' defect on bone scintigraphy as well as FDG PET/CT. ${ }^{6-9}$ Uptake of MDP is extremely rare in a hemangioma, and the role of SPECT/CT in the diagnosis of this rare phenomenon has been reported only once. ${ }^{10,11}$ While two of the multiple hemangiomas in the present case showed MDP uptake, all other lesions were relatively photopenic compared to the background. Absence of FDG uptake in these lesions may be due to the lack of any metabolically active tissue in hemangiomas. This case demonstrates the variation of MDP uptake in hemangiomas and emphasizes the need for correlation of whole body planar scintigraphy with anatomical imaging.

\section{REFERENCES}

1. Even-Sapir E. Imaging of malignant bone involvement by morphologic, scintigraphic, and hybrid modalities. J Nucl Med 2005;46:1356-67.

2. Gnanasegaran G, Barwick T, Adamson K, Mohan H, Sharp D, Fogelman I. Multislice SPECT/CT in benign and malignant bone disease: When the ordinary turns into the extraordinary. Semin Nucl Med 2009;39:431-42.
3. Sharma P, Singh H, Kumar R, et al. Bone scintigraphy in breast cancer: Added value of hybrid SPECT-CT and its impact on patient management. Nucl Med Commun 2012;33:139-47.

4. Franc BL, Myers R, Pounds TR, et al. Clinical utility of SPECT(Low-Dose) CT versus SPECT alone in patients presenting for bone scintigraphy. Clin Nucl Med 2012;37:26-34.

5. Fox MW, Onofrio BM. The natural history and management of symptomatic and asymptomatic vertebral hemangiomas. J Neurosurg 1993;78:36-45.

6. Makhija M, Boll ER. Hemangioma, a rare cause of photopenic lesion on skeletal imaging. Clin Nucl Med 1988;13:661-62.

7. Sopov V, Liberson A, Gorenberg M, Groshar D. Cold vertebrae on bone scintigraphy. Semin Nucl Med 2001;31:82-83.

8. Reader DW, Pozderac RV. Vertebral hemangioma presenting as a cold defect on bone scintigraphy. Clin Nucl Med 2001;26: 868-69.

9. Domínguez M, Rayo J, Serrano J, et al. Vertebral hemangioma: Cold vertebrae on bone scintigraphy and fluordeoxyglucose positron emission tomography-computed tomography. Indian J Nucl Med 2011;26:49-51.

10. Halkar RK, Motawy MM, Hebbar HG, Jahan MS. Vertebral body hemangioma showing increased uptake of Tc-99m MDP and decreased Tc-99m labeled red blood cells. Clin Nucl Med 1994;19:827-28.

11. Bhoil A, Mittal BR, Harisankar CN, Bhattacharya A, Singh P, Aggarwal S. Methylene diphosphonate uptake in a case of vertebral hemangioma: Demonstration with hybrid SPECT/CT. Clin Nucl Med 2011;36:65-66.

\section{ABOUT THE AUTHORS}

\section{K Manohar}

Senior Resident, Department of Nuclear Medicine, Postgraduate Institute of Medical Education and Research, Chandigarh, India

\section{RVR Parghane}

Junior Resident, Department of Nuclear Medicine, Postgraduate Institute of Medical Education and Research, Chandigarh, India

\section{S Kumari}

Senior Resident, Department of Nuclear Medicine, Postgraduate Institute of Medical Education and Research, Chandigarh, India

\section{A Bhattacharya (Corresponding Author)}

Additional Professor, Department of Nuclear Medicine Postgraduate Institute of Medical Education and Research Chandigarh, India, Phone: 91-172-2756726 (O), 91-9872486816 (M) Fax: 91-172-2744401, e-mail: anishpgi@yahoo.co.in

\section{AK Mandal}

Professor and Head, Department of Nuclear Medicine and Urology Postgraduate Institute of Medical Education and Research Chandigarh, India

\section{BR Mittal}

Professor and Head, Department of Nuclear Medicine, Postgraduate Institute of Medical Education and Research, Chandigarh, India 